\title{
Flow associated or flow mediated dilatation? More than just semantics
}

When blood flow increases through a blood vessel the vessel dilates. This phenomenon-flow dependent dilatation-has been demonstrated in a number of vessels in vitro $^{1}$ and in vivo, ${ }^{2}$ in animals and humans. ${ }^{3}$ Flow dependent dilatation appears to be an autoregulatory process that might oppose classical Bayliss-type pressure dependent contraction (the process by which a vessel constricts if the intraluminal pressure rises). ${ }^{4}$ In most ${ }^{12}$ but not all vessels studied, ${ }^{5}$ flow dependent dilatation was contingent on the presence of an intact endothelial layer. The endothelium appears to act as a mechanotransducer, sensing changes in shear stress and modifying the output of dilator factors accordingly. ${ }^{6}$ The precise sensing mechanism is not known, but stretch activated cation channels, ${ }^{7}$ shear stress activated potassium channels, ${ }^{8}$ and sodium channels ${ }^{9}$ in the endothelial cell membrane have all been implicated. The major effector of flow mediated dilatation appears to be endothelium derived nitric oxide. ${ }^{10}$ The physiological role of flow dependent dilatation is not known, but it is possible that it is a mechanism to keep shear stress constant in the face of alterations in the volume of blood delivered to tissues. ${ }^{11}$

It was on the background of these basic studies that Celermajer and colleagues ${ }^{12}$ devised an ingenious method to study endothelial function in humans. They postulated that an increase in flow through the brachial artery should be sufficient to induce dilatation of the vessel, and that the degree of dilatation should be an indication of the functional state of the endothelium. Increase in flow through the brachial artery was induced by causing post-ischaemic dilatation in a downstream vascular bed-the hand. This was achieved by inflating to suprasystolic pressure a cuff placed around the wrist to produce ischaemia in the hand, and then releasing the pressure in the cuff to restore flow into the dilated bed. The subsequent increase in flow through the brachial artery caused a dilatation of this vessel that was assessed using an ultrasonic device to detect the vessel wall. This technique has been widely adopted as a non-invasive means to explore endothelial function, and changes in flow dependent dilatation have been associated with virtually all of the major cardiovascular risk factors. ${ }^{13} 14$ As a control, the response of the brachial artery to sublingual glyceryl trinitrate was recorded.

\section{Stimulus for dilatation}

Dilatation of the brachial artery occurs after flow is increased, but is the increased flow the stimulus for the dilatation? Leeson and colleagues measured flow and brachial artery diameter simultaneously and, as reported in this issue, ${ }^{15}$ they showed that, following hand ischaemia, the increase in blood flow through the brachial artery was immediate and short lived, whereas the subsequent dilatation of the artery was slow in onset, progressive (it reached a peak up to two minutes after cuff deflation), and persistent (it lasted for 10 to 20 minutes). In other words, the dilatation of the brachial artery was maximal after blood flow has returned to baseline values. Similar results have been reported by Corretti et al. ${ }^{16}$

These observations raise the question of whether flow really is the stimulus initiating the dilatation of the brachial artery that seems to occur during the recovery from hand ischaemia. One possibility is that the biochemical changes which result from transient endothelial exposure to increased flow can in some way induce sustained changes in the endothelial nitric oxide pathway that persist after the flow stimulus is removed. Alternatively, it is possible that some metabolite washed out of the ischaemic hand contributes to the phenomenon, perhaps by activating perivascular nerves. Until these complex issues are resolved, it may be preferable to refer to the observed vascular response in the brachial artery as flow associated rather than flow mediated dilatation.

\section{Role of the endothelium}

In most vessels an intact endothelium is a prerequisite for flow dependent dilatation. However, this is not a universal finding and in some vessels flow dependent constriction or dilatation may occur in vessels denuded of endothelium. ${ }^{5}$ It is not possible to test directly whether the response of the brachial artery is endothelium dependent, but studies in a similar sized vessel, the femoral artery of the $\mathrm{dog},{ }^{2}$ clearly demonstrated that an intact endothelium was essential. It has also been demonstrated that flow associated dilatation in the brachial artery is abolished by the nitric oxide synthase inhibitor L-NMMA. ${ }^{18}{ }^{18}$ While it is possible that the nitric oxide derives from nerves, the simplest interpretation of the experimental findings is that endothelium derived nitric oxide mediates the flow associated dilatation of the human brachial artery. Impairment of flow associated dilatation of the brachial artery appears to correlate with a diminished response to endothelium dependent dilators in other vascular beds, including the coronary circulation, suggesting that the measure has some validity as a non-invasive surrogate of endothelial function.

\section{Glyceryl trinitrate as a control}

A preserved response of the brachial artery to sublingual glyceryl trinitrate or other nitrates, in the face of an impaired flow associated dilatation, is usually interpreted as evidence for a specific defect in endothelial function. However, the dose of nitrate used is often sufficient to produce significantly greater dilatation than that seen as flow associated dilatation. Typically flow associated dilatation is in the order of $6 \%$ whereas nitrate induced dilatation is in the order of $15 \%$. Assuming that both responses are mediated by nitric oxide, one from an endogenous source the other exogenous, the interpretation of similar or different responses may not be entirely straightforward. Indeed, if the smooth muscle is in some way insensitive to the actions of nitric oxide, or if the nitric oxide is more rapidly destroyed in disease states, it would be possible to see a 


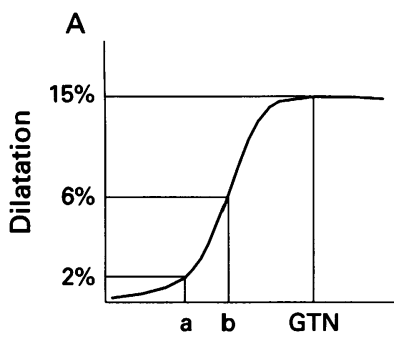

B

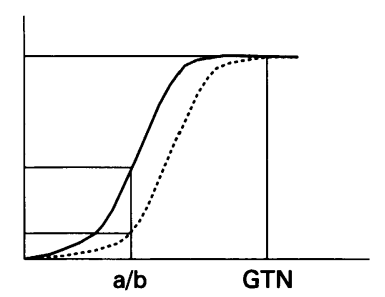

Nitric oxide concentrations produced by flow or glyceryl trinitrate (GTN). (A) Standard interpretation. Diseased endothelium (a) generates less bioactive nitric oxide in response to flow and this results in less dilatation. The dilatation in response to GTN remains unchanged in healthy and diseased vessels, and the dose used produces maximal dilatation in both conditions. (B) Alternative interpretation. Diseased endothelium generates equivalent bioactive nitric oxide in response to flow (a), but the smooth muscle response to nitric oxide is reduced (broken line), resulting in a rightward shift of the curve. The resultant dilatation to flow is reduced in the diseased vessel. The response to GTN appears unchanged as the dose used is supramaximal and produces a maximal dilatation in both healthy and diseased vessels. Use of lower doses of GTN to construct a dose-response relationship might unmask such differences.

picture of responses that is indistinguishable from that produced by endothelial dysfunction (fig).

Even using a high dose, the dilator response to nitrates appears to be attenuated in certain disease states, ${ }^{19}$ suggesting that reduced sensitivity to released nitric oxide might contribute to the reduction in flow associated dilatation. This means that the defect might not lie at the level of the endothelium. An alternative explanation is that part of the response to nitrates is also flow mediated (these drugs will increase flow in the arm), and it is this part of the response which is lost in the presence of cardiovascular disease.

\section{Summary}

Dilatation of the brachial artery occurs after flow is increased, and an attenuation in this response is seen in subjects with cardiovascular risk factors, and in those with established coronary artery disease. The mechanisms linking ischaemia, flow changes, and brachial artery dilatation are unclear, and it is not known how these are affected by arterial disease. For the present it might be more appropriate to refer to flow associated rather than flow mediated dilatation, to describe the phenomenon in the brachial artery.

Despite these caveats, the non-invasive measurement of brachial artery following ischaemic dilatation represents a significant advance, and its suitability as a surrogate marker for coronary artery dysfunction appears promising. The technique has potential as a tool for screening those at high risk of vascular disease, and as a surrogate end- point in intervention studies. Further research should clarify the mechanisms involved, and lead to greater insights into the nature of endothelial dysfunction and cardiovascular disease.

Kiran Bhagat and Aroon Hingorani are supported by the British Heart Foundation

KIRAN BHAGAT AROON HINGORANI PATRICK VALLANCE

Centre for Clinical Pharmacology,

Cruciform Project for Strategic Medical Research, and

Department of Medicine,

University College London,

London WC1E 6FY, United Kingdom

e-mail: k.bhagat@ucl.ac.uk

1 Kuo L, Davies MJ, Chilian WM. Endothelium-dependent, flow-induced dilation of isolated coronary arterioles. Am f Physiol 1990;259: H1063-70.

2 Pohl U, Holtz J, Busse R, Bassenge E. Crucial role of the endothelium in the vasodilator response to flow in vivo. Hypertension 1985;8:37-44.

3 Drexler $\mathrm{H}$, Zeiher AM, Wollschlager $\mathrm{H}$, Meinertz T, Just $\mathrm{H}$, Bonzel $T$. Flow-dependent coronary artery dilatation in humans. Circulation 1989; 80:466-74

4 Baylis WM. On the local reaction of the vessel wall to changes of internal pressure. F Physiol 1902;28:220-31.

5 Bevan JA, Joyce EH, Wellmann GC. Flow-dependent dilation in a resistance artery still occurs after endothelium removal. Circ Res 1988;63: $980-5$.

6 Davies PF. Flow-mediated endothelial mechanotransduction. Physiol Rev 1995;75:519-59.

7 Lansman JB, Hallam TJ, Rink TJ. Single stretch-activated ion channels in vascular endothelial cells as mechanotransducers. Nature 1987;235: 811-13.

8 Olsen SP, Clapham DE, Davies PF. Haemodynamic shear stress activates a $\mathrm{K}^{+}$current in endothelial cells. Nature 1988;331:168-70.

9 Siegel G, Malmsten M, Klussendorf D, Walter A, Schnalke F, Kauschmann A. Blood-flow sensing by anionic biopolymers. $\mathcal{F}$ Auton Nerv Syst 1996;57:207-13.

10 Pohl U, Herelan K, Huang A, Bassenge E. EDRF-mediated shear-induced dilatation opposes myogenic vasocontriction in small rabbit arteries. $A m \mathcal{F}$ dilatation opposes myogenic

11 MacAllister RJ, Vallance, P. Systemic vascular adaptation to increases in blood volume: the role of the blood vessel wall. Nephrol Dial Transplant 1996;11:231-40.

12 Celermajer DS, Sorensen KE, Gooch VM, Spiegelhalter DJ, Miller OI, Sullivan ID, et al. Non-invasive detection of endothelial dysfunction in children and adults at risk of atherosclerosis. Lancet 1992;340:1111-15.

13 Celermajer DS, Sorensen KE, Georgakopoulos D, Bull C, Thomas O, Robinson J, et al. Cigarette smoking is associated with dose related and potentially reversible impairment of endothelium-dependent dilation in healthy young adults. Circulation 1993;88:2149-55.

14 Celermajer DS, Sorensen KE, Bull C, Robinson J, Deanfield JE. Endothelium-dependent dilation in the systemic arteries of asymptomatic subjects relates to coronary risk factors and their intervention. $\mathcal{f} \mathrm{Am}$ Coll Cardiol 1994;24:1468-74.

15 Leeson P, Thorne S, Donald A, Mullen M, Clarkson P, Deanfield J. Noninvasive measurement of endothelial function: effect on brachial artery invasive measurement of endothelial function: effect on brachial artery Heart 1997;78:22-7.

16 Corretti MC, Plotnick GD, Vogel RA. Technical aspects of evaluating brachial artery dilation using high frequency ultrasound. Am $\mathcal{f}$ Physiol 1995;268:H1397-404

17 Lieberman EH, Knab ST, Creager MA. Nitric oxide mediates the vasodilator response to flow in humans [abstract]. Circulation 1994;90:I138.

18 Joannides R, Haefeli WE, Linder L, Richard V, Bakkali EH, Thuillez C, et al. Nitric oxide is responsible for flow-dependent dilatation of human peripheral conduit arteries in vivo. Circulation 1995;91:1314-19.

19 Thorne SA, Clarkson P, Mullen MJ, Donald AE, Thomson H, Powe A, et al. Early endothelial dysfunction in insulin-dependent diabetes mellitus and hypercholesterolaemia: response to L-arginine [abstract]. Circulation 1996;94:1702. 